\title{
Inversión extranjera directa y desigualdad de los ingresos en América Latina. Un análisis sectorial
}

\author{
Macarena Suanes
}

RESUMEN

En este trabajo se analiza la relación entre la inversión extranjera directa (IED) y la desigualdad de los ingresos en América Latina. En particular, se estima el efecto de la IED desde una perspectiva sectorial y se identifican tres grandes sectores: primario, industria manufacturera y servicios. Mediante un panel de datos para 13 economías en el período 1980-2009, se encontró evidencia empírica de un efecto positivo de la IED en la desigualdad de los ingresos provocada por aquellas inversiones localizadas en los sectores de servicios e industria manufacturera. 


\section{I}

\section{Introducción}

A partir de la década de 1990, América Latina se ha convertido en uno de los principales destinos de la inversión extranjera directa (IED), según datos de la Conferencia de las Naciones Unidas sobre Comercio y Desarrollo (UNCTAD, 2011). En efecto, durante el período 1995-2009, la IED en términos del producto interno bruto (PIB) se triplicó con creces con respecto a los niveles verificados en la década de 1980. Este proceso se ha visto acompañado en la región de un crecimiento económico significativo (especialmente en el quinquenio 2004-2009 con una expansión del 5\% promedio anual) y de una elevada y persistente desigualdad de los ingresos. Este último fenómeno se ha transformado en una de las principales preocupaciones de los responsables de la política económica, no solo por su relevancia, sino también por su persistencia en el tiempo.

Si bien la mayoría de la literatura que se ha centrado en estudiar los efectos de la IED en el crecimiento económico apunta a un impacto positivo de esta en el crecimiento económico en las economías en desarrollo (De Mello, 1997 y 1999; Borensztein, De Gregorio y Lee, 1998; Li y Liu, 2005; Herzer, Klasen y Nowak-Lehmann, 2008; De Vita y Kyaw, 2009), la relación entre la IED y la desigualdad de los ingresos no resulta tan evidente y ha recibido menos atención en la literatura. Así, en numerosos trabajos en los que se estima la relación entre la IED y la desigualdad de los ingresos, se halla una relación positiva (Tsai, 1995; Velde, 2003; Choi, 2006; Basu y Guariglia, 2007; Herzer, Hühne y Nunnenkamp, 2012), mientras que en otros trabajos no se logra verificar la existencia de una relación entre estas dos variables o se encuentra una relación negativa (Milanovic, 2003; Sylwester, 2005). A nivel microeconómico, sin embargo, existe una amplia rama de la literatura en la que se halla que - en presencia de la IED — la brecha salarial entre los trabajadores calificados y no calificados aumenta y, por lo tanto, también la desigualdad de los ingresos (Lipsey y Sjöholm, 2004; Mah, 2002; Velde, 2003; Aitken, Harrison y Lipsey, 1996; Feenstra y Hanson, 1997). En consecuencia, si bien no existe consenso, pareciera que la evidencia empírica apunta a una mayor desigualdad de los ingresos provocada por los flujos de la IED.

Otro aspecto que puede resultar relevante para analizar el efecto de la IED en la desigualdad de los ingresos y que ha recibido escasa atención en la literatura, se refiere a cómo puede afectar la distribución sectorial de dicha inversión. La IED tiende a localizarse en distintos sectores económicos dependiendo de las características y atractivos que presenta cada economía, como serían la abundancia de mano de obra barata, la abundancia de recursos naturales y los beneficios fiscales o institucionales. En este sentido, la hipótesis planteada en este estudio es que los canales por los que la IED puede afectar a la desigualdad no serían independientes de los sectores de la economía en los que esa inversión se localiza en cada país.

De hecho, la distribución sectorial de la IED en las economías de América Latina es muy dispar según el país que se considere. De acuerdo con datos de la UNCTAD (2006), en el año 2006 la industria manufacturera representó en promedio el $26 \%$ del total de la IED en la región, concentrándose en los países más desarrollados, como la Argentina, Chile, Costa Rica y México. En muchos otros países latinoamericanos, los sectores de la minería y el petróleo representan una cuota importante del total de la IED, tal sería el caso de Bolivia (Estado Plurinacional de), Chile y Colombia, donde en 2006 dichos sectores concentran entre la mitad y la tercera parte de la IED total. Por otra parte, cabe destacar que la IED en el sector agrícola es insignificante en la mayoría de los países de la región. Finalmente, el sector de servicios ha visto aumentado su protagonismo en forma generalizada en los últimos años concentrando a nivel agregado de América Latina el 50\% del total de la IED, con especial protagonismo de los servicios financieros y del transporte y las telecomunicaciones, así como de la distribución de electricidad, gas y agua.

Si bien no hay evidencia empírica a nivel macroeconómico sobre el impacto de la IED según sectores en la desigualdad de los ingresos en las economías en desarrollo, y en particular en la región, sí existe evidencia del efecto de la IED por sectores en el crecimiento económico y la productividad. Así, Tondl y Fornero (2010) encuentran evidencia de un efecto positivo de la IED en la productividad en todos los sectores de actividad de las economías de América Latina, siendo más elevados los efectos en los sectores primario y de servicios financieros.

En este contexto, en este artículo se analiza y aporta evidencia empírica acerca del impacto que la IED por 
sectores tiene en la desigualdad de ingresos para el caso de los países de América Latina. La escasa literatura que estudia el efecto de la IED en la desigualdad de ingresos en la región, junto con las principales tendencias observadas en esta durante la última década (importante crecimiento económico, incremento en los flujos de IED y persistente desigualdad de los ingresos) son la principal motivación del presente estudio, que aporta como contribución más novedosa el hecho de permitir identificar los efectos de la IED según los sectores en que se localiza.

Para alcanzar este objetivo, en este trabajo se analizarán dos escenarios; un primer escenario en que se considera la relación de la IED a nivel agregado con la desigualdad de los ingresos, y un segundo escenario en que se analiza la relación de la IED, especificando los principales sectores de la economía en los que se localiza (primario, industria manufacturera y servicios) y la desigualdad de los ingresos.

En el gráfico 1 se ilustra para las economías de América Latina la relación de la IED como porcentaje del PIB con la desigualdad de los ingresos (medida con el coeficiente de Gini), tomando las medias de dichas variables respecto del período 1980-2009. En el gráfico 1 se aprecia intuitivamente una relación positiva entre ambas variables.

En cambio, cuando en el gráfico 2 se visualiza la relación entre la IED desagregada por sectores de actividad y la desigualdad de los ingresos, la relación presenta matices según el sector al que se hace referencia. De este modo, mientras que el sector primario y el sector servicios sí parecen sugerir una relación positiva, el sector de la industria no parece mostrar una relación clara.

El resto del trabajo se organiza como sigue. En la sección II se presenta una breve revisión de la literatura sobre la relación entre la IED y la desigualdad de los ingresos, con especial énfasis en el análisis sectorial. En la sección III se muestra la descripción de los datos y de las principales tendencias de las variables de interés en la región. En la sección IV se expone la estrategia empírica, mientras que la discusión de los resultados se halla en la sección V. Por último, el documento concluye en la sección VI.

GRÁFICO 1

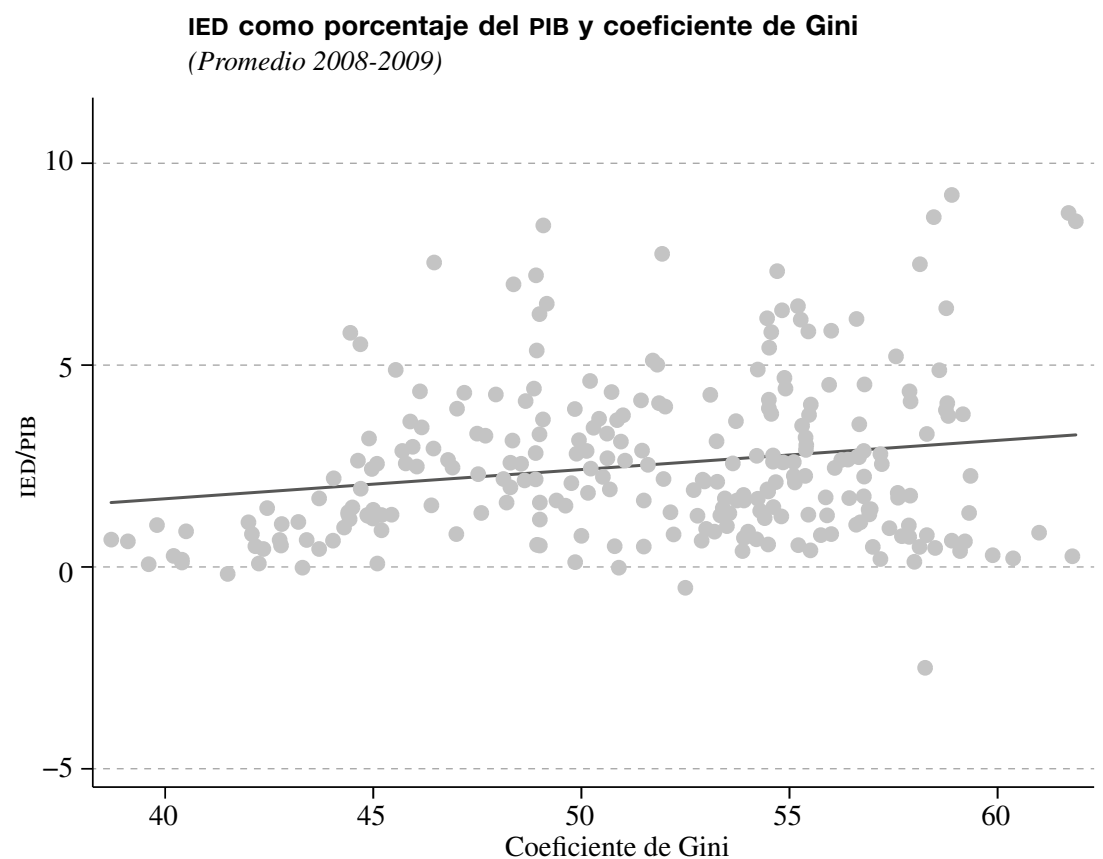

Fuente: Elaboración propia mediante información de la Base de datos sobre la desigualdad de los ingresos en el mundo (WIID) e Indicadores del Desarrollo Mundial (WDI).

Nota: IED: Inversión extranjera directa; PIB: Producto interno bruto. 
GRÁFICO 2

Coeficiente de Gini e IED por sectores de actividad (IED en términos del PIB sectorial)

Promedio 2008-2009

a. Sector primario

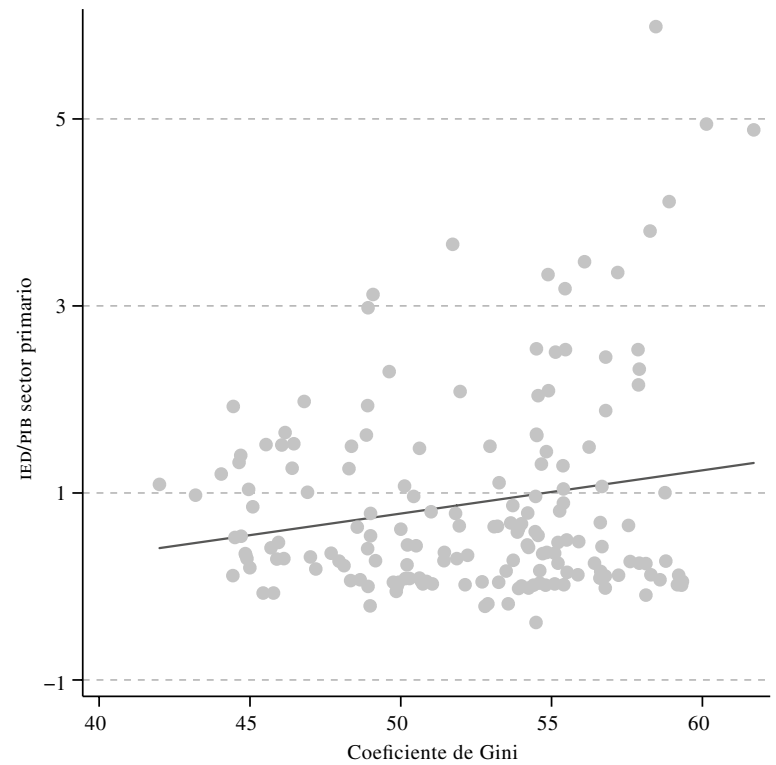

b. Industria manufacturera

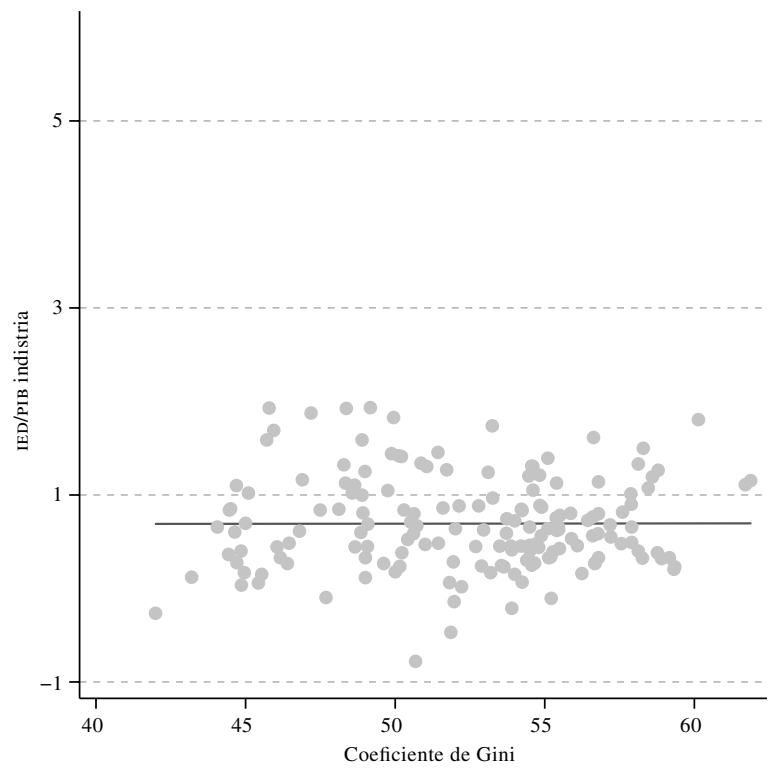

c. Sector servicios

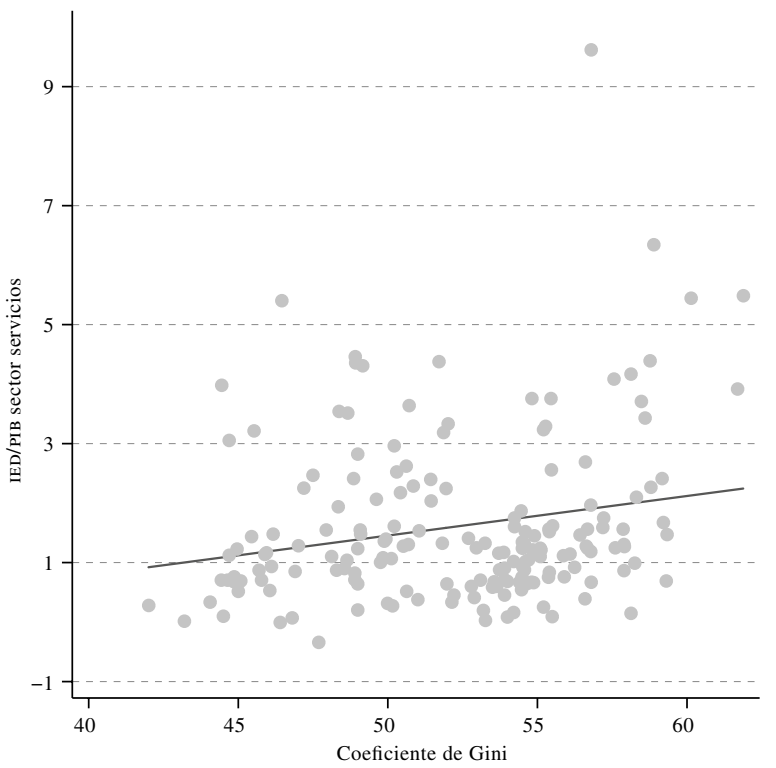

Fuente: Elaboración propia mediante información de la Base de datos sobre la desigualdad de los ingresos en el mundo (wIID). Nota: IED: Inversión extranjera directa; PIB: Producto interno bruto. 


\section{II}

\section{Revisión de la literatura}

En esta sección se presenta una revisión somera de la literatura tanto teórica como empírica, enfocada, por una parte, en la relación de la IED y la desigualdad de los ingresos, y por otra, en el efecto de la IED en la desigualdad de los ingresos, identificando los principales sectores de actividad en los que esa inversión se localiza.

\section{Inversión extranjera directa (IED) y desigualdad de los ingresos}

La relación entre el crecimiento económico y la IED ha recibido más atención por parte de la literatura durante la década de 1990, debido al importante proceso de liberalización del comercio que experimentaron las economías en desarrollo en este período. Así, la literatura empírica presenta un relativo consenso en relación con el impacto positivo de la IED en el crecimiento económico en los países en desarrollo (De Mello, 1997 y 1999; Borensztein, De Gregorio y Lee, 1998; Li y Liu, 2005; Herzer, Klasen y Nowak-Lehmann, 2008; De Vita y Kyaw, 2009). En cambio, el efecto de la IED en la desigualdad de los ingresos, especialmente en las economías en desarrollo, ha recibido menos atención debido muy probablemente a la limitación en los datos y a la escasa literatura existente que estudia los posibles vínculos teóricos entre ambas variables.

A continuación se detallan las principales contribuciones relativas a los posibles canales de impacto que presenta la IED respecto de la desigualdad de los ingresos. Así, Jensen y Rosas (2007) sugieren dos canales a través de los cuales esta inversión puede afectar a la desigualdad. En primer lugar, la IED aporta capital al país, disminuyendo así la rentabilidad total para el capital y aumentando los rendimientos de la mano de obra. Por lo tanto, el capital extranjero compite con el capital nacional por captar a los trabajadores domésticos, acrecentando los salarios y aminorando la rentabilidad de las empresas nacionales. Este efecto tendería a reducir la desigualdad de los ingresos a través de la disminución de la brecha entre rentas salariales y rentas del capital. La segunda vía por la que la IED puede tener un impacto en la desigualdad, es que las empresas extranjeras tienden a pagar una prima salarial especialmente en el caso de los trabajadores calificados, dando lugar a un incremento de la brecha de ingresos entre trabajadores calificados y no calificados, y por consiguiente, a un aumento de la desigualdad. Ahora bien, si estas empresas extranjeras pagaran también una prima salarial a los trabajadores no calificados, la IED contribuiría a reducir la desigualdad de los ingresos mediante el incremento de los ingresos de los trabajadores de menores recursos.

Por su parte, Velde (2003) recogiendo otras aportaciones, se refiere a tres posibles canales por los que la IED puede afectar a la desigualdad salarial en los países en desarrollo. En primer lugar, identifica un "efecto composición" como resultado de que las firmas extranjeras tienden a localizarse en sectores más intensivos en mano de obra calificada, mejorando así la posición de estos trabajadores con respecto a los no calificados (Feenstra y Hanson, 1997). En segundo lugar, la IED también puede afectar a la oferta de trabajadores calificados a través de la capacitación y las contribuciones específicas a la educación general (transferencia de conocimientos). Y por último, en términos de Berman, Bound y Machin (1998), la IED puede probablemente inducir un crecimiento más rápido de la productividad laboral tanto en empresas extranjeras (transferencia de tecnología), como en empresas nacionales (efectos secundarios), y si el crecimiento de la productividad está sesgado a sectores calificados, entonces aumentará la brecha entre estos sectores. En este sentido, Velde (2003), mediante un análisis transversal (cross-section) para cuatro economías de América Latina (Chile, Colombia, Bolivia (Estado Plurinacional de) y Costa Rica) en el período 1978-2000, encuentra evidencia empírica en favor de que la IED incrementa la desigualdad salarial en la región y por esta vía la desigualdad de los ingresos. Además, en varios estudios de países se apoya la hipótesis de que la IED, principalmente en las economías en desarrollo, se vincula a una mayor desigualdad en los ingresos salariales. Algunos ejemplos en este sentido son los casos de Indonesia (Lipsey y Sjöholm, 2004), la República de Corea (Mah, 2002) y México (Aitken, Harrison y Lipsey, 1996; Feenstra y Hanson, 1997). Aunque hay que poner de relieve que gran parte de la evidencia empírica permite ver también que la IED se relaciona con mayores salarios para todos los tipos de trabajadores (Instituto de Desarrollo de Ultramar, 2002).

Sin embargo, la literatura empírica en que se estudia la relación entre la IED y la desigualdad a nivel 
macroeconómico no es concluyente. Así, Tsai (1995), mediante un análisis de sección cruzada para 53 economías estima una relación positiva y significativa entre la IED y la desigualdad, aunque cuando controla por variables ficticias (América Latina y Asia) encuentra que la relación positiva podría estar capturando las diferencias en desigualdad entre economías, más que el papel que estaría jugando la IED. Por su parte, Choi (2006), por medio de un panel de 119 países en el período 19932002, obtiene evidencia en favor de que un incremento en la IED aumenta la desigualdad. En la misma dirección apunta el trabajo de Basu y Guariglia (2007) quienes, utilizando un panel de 119 economías en desarrollo respecto del período 1970-1999, reportan una relación positiva entre la IED, el crecimiento económico, la desigualdad de los ingresos y el capital humano. Además, en un trabajo reciente, Herzer, Klasen y Nowak-Lehmann (2008), mediante técnicas de cointegración analizan el impacto de la IED en la desigualdad de los ingresos de largo plazo para cinco economías de América Latina (Bolivia (Estado Plurinacional de), Chile, Colombia, México y Uruguay), mostrando que la IED tiene un efecto significativo y positivo en la desigualdad de los ingresos en casi todas estas economías. En contraste, Milanovic (2003), empleando datos de encuestas de hogares para 129 países en los años 1988, 1993 y 1998, encuentra que la IED no presenta efectos en la distribución del ingreso. Finalmente, Sylwester (2005), utilizando un panel de 29 economías en desarrollo para el período 1970-1990, no halla evidencia de una relación positiva entre estas variables. En resumen, si bien la evidencia empírica relativa a la relación entre la IED y la desigualdad en las economías en desarrollo no es concluyente, en ningún caso apunta hacia un efecto de reducción en la desigualdad, sino más bien al contrario.

\section{La inversión extranjera directa (IED) por sectores y la desigualdad de los ingresos}

En la literatura que estudia la relación entre la IED y la desigualdad de los ingresos no se ha prestado atención a un aspecto que puede ser relevante para entender el impacto de la IED en las economías en desarrollo, como es capturar el efecto de la composición sectorial de la IED. En este sentido, la hipótesis es que cabría esperar que la IED no tenga el mismo impacto independientemente del sector de la economía que reciba dicha inversión, efecto que el análisis agregado no permite determinar. Por lo tanto, los canales a través de los que la IED afecta a la desigualdad es probable que sean diferentes según el sector receptor de esa inversión.
De esta manera, en la literatura se identifican algunos efectos diferenciales de la IED en la desigualdad de los ingresos según los sectores económicos. El sector de la industria manufacturera suele ser intensivo en mano de obra, y las empresas extranjeras tienden a invertir en este tipo de sectores en los países en desarrollo en busca de mano de obra barata y reducción de sus costos. De hecho, la mayoría de las teorías sobre el efecto distributivo de la IED se refieren implícitamente al caso de las inversiones en la industria manufacturera intensiva en trabajo. La conclusión a la que arriban es que la IED reduce la desigualdad de los ingresos en economías con mano de obra abundante con salarios bajos al aumentar la demanda de trabajadores no calificados y ofrecer salarios más altos que los que prevalecen en la economía doméstica. Según esta perspectiva, la IED mejoraría la distribución de ingresos en los países receptores de IED (Cornia, 2011). Sin embargo, la evidencia empírica no es concluyente en este sentido y no logra demostrarlo. Por ejemplo, Velde y Morrissey (2004) encontraron que la IED aumentó los salarios de todos los niveles de calificación en cuatro de los cinco países de Asia Oriental analizados. En cambio, en México, el incremento de los salarios en presencia de la IED fue significativamente menor para los trabajadores no calificados en relación con los cualificados (Alarcón y McKinley, 1996).

Por otra parte, en el sector primario la IED tiende a exacerbar la desigualdad, ya que la propiedad de los recursos naturales se concentra en pocas manos y las rentas resultantes son capturadas por los que se encuentran en el extremo superior de la distribución. Por último, el sector servicios no se puede clasificar como un sector intensivo en trabajo o capital, de modo que su efecto en la desigualdad no es tan evidente. Para analizar el efecto de los flujos de capital de este sector es necesario estudiar los subsectores. Por ejemplo, las telecomunicaciones son intensivas en capital y utilizan mano de obra calificada, mientras que, por ejemplo, el sector del turismo es más intensivo en mano de obra no calificada. De esta forma, se puede esperar que, en el primer caso, la IED aumente la desigualdad, mientras que en el segundo caso la disminuya (Cornia, 2004).

En los estudios empíricos a nivel macroeconómico no se suele identificar el efecto de la IED en la desigualdad según sectores económicos. Sin embargo, otras ramas de la literatura sí incluyen en el análisis la desagregación de la IED por sectores. Por ejemplo, Aykut y Sayek (2007) encuentran que la composición sectorial de la IED sí importa para explicar el crecimiento de la productividad en los países en desarrollo y concluyen 
que una alta participación de la agricultura en el total de la IED tiene un efecto negativo para el crecimiento de una economía, mientras que una alta participación de la IED en el sector manufacturero tiene un efecto positivo. Por su parte, Nunnenkamp y Spatz (2004) hallan que el crecimiento agregado es mayor si un país tiene una elevada cuota de la IED en la industria de la maquinaria y equipos eléctricos, en lugar de las industrias alimentaria, química y metalúrgica. Asimismo, Tondl y Fornero (2010) indican efectos positivos de la IED en la productividad en todos los sectores de las economías latinoamericanas, a pesar de que pueden depender de condiciones específicas o se limitan a un cierto período de tiempo. Los efectos directos de la productividad son más elevados en el sector primario (agricultura, minería y producción de petróleo) y en los servicios financieros.

\section{III}

\section{Datos}

Para estimar el impacto de la IED en la desigualdad de los ingresos por sectores, se construyó un panel de datos no balanceado respecto del período 1980-2009 para 13 economías de América Latina. Los países considerados en la muestra son: Argentina, Bolivia (Estado Plurinacional de), Brasil, Chile, Colombia, Costa Rica, Ecuador, Honduras, México, Paraguay, Perú, República Dominicana y Uruguay. La elección de los países se ha determinado de acuerdo con la disponibilidad de datos de la IED por sectores. A su vez, se consideraron dos aproximaciones temporales utilizando datos anuales y también datos trianuales. Dicha doble aproximación se explica porque la utilización de datos anuales permite maximizar la limitada disponibilidad de datos existentes, mientras que el uso de datos trianuales también se consideró debido a que, por una parte, no se esperan cambios importantes año a año en algunas de las variables macroeconómicas, como es el caso de la desigualdad; y por otra, así se reducen las fluctuaciones a corto plazo y por lo tanto la influencia del ciclo económico, lo que permite centrar la atención en la relación de largo plazo.

Como medida de inversión extranjera directa se utiliza la entrada neta de capital extranjero en términos de PIB. Los datos agregados se obtuvieron a partir de la base de datos de Indicadores del Desarrollo Mundial (WDI, por sus siglas en inglés). Como se puede apreciar en el gráfico 3, en el segundo subperíodo analizado (1995-2009), la IED se triplicó con creces respecto del

GRÁFICO 3

América Latina (13 países): inversión extranjera directa (IED) (En porcentajes del PIB)

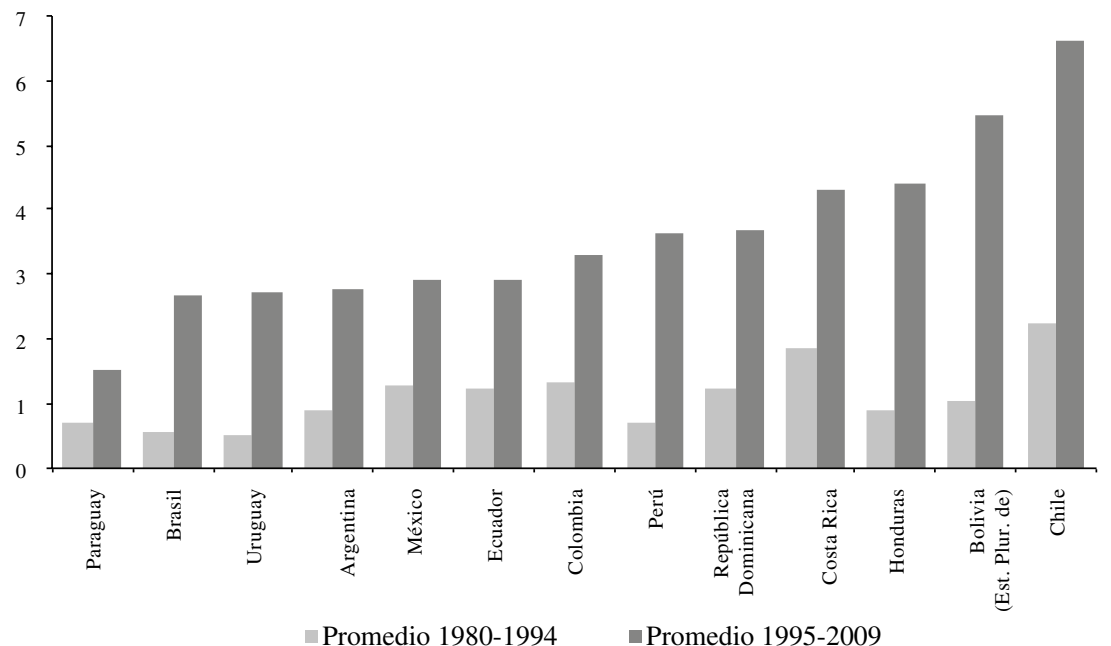

Fuente: Elaboración propia sobre la base de Indicadores del Desarrollo Mundial (WDI).

Nota: PIB: Producto interno bruto. 
período anterior (1980-1994). Ello se debió, por una parte, al proceso de liberalización iniciado a principios de los años noventa en la región, proceso que además estuvo acompañado de grandes reformas y amplios programas de privatización de empresas públicas y de servicios en varios países de la región. Y por otra parte, a las pronunciadas caídas de las tasas de interés observadas entre 2002 y 2008 , que incentivaron a las economías con cuantiosa acumulación de capitales a salir en busca de inversiones más rentables, como por ejemplo, en Asia y América Latina. Dentro de las economías que más IED reciben en el segundo período se destacan Chile, Bolivia (Estado Plurinacional de) y Honduras, mientras que el Paraguay, el Brasil y el Uruguay presentan la razón de IED más baja en términos del PIB. Sin embargo, en términos absolutos, el Brasil y México siguen siendo las economías que, de manera muy destacable, más flujos de IED reciben en millones de dólares (véase el cuadro A.1 del anexo). Asimismo, si se compara con otras regiones se comprueba que América Latina, junto con Asia, es de las regiones de las economías en desarrollo que más flujos de IED reciben y donde más ha crecido dicha inversión en el período analizado (véase el cuadro A.2 del anexo).

$\mathrm{Al}$ igual que para la IED a nivel agregado, para la IED por sectores de actividad se utilizaron los flujos netos por sectores en términos del PIB. Las fuentes de datos para la IED sectorial varían según el país, ya que no existe esfuerzo por parte de ninguna institución en sistematizar una serie de datos de IED por sectores de actividad $^{1}$. En general, las fuentes que se han utilizado en este trabajo para la elaboración de dichas series de datos son los bancos centrales de cada país, los institutos de estadísticas y, en algunos casos, institutos u organizaciones creadas específicamente con el fin de promover este tipo de inversión (véase el cuadro A.5 del anexo). Debido a la gran heterogeneidad de información disponible por países y con el objetivo de poder elaborar una serie de datos lo más homogénea y comparable posible, se decidió agrupar los datos en tres grandes sectores: sector primario (incluye agricultura, minería e industria extractiva), industria manufacturera y servicios.

Como se puede observar en el gráfico 4, tomando medias del período 1980-2009, el sector más importante para explicar la evolución de la IED en la región es el sector servicios que explica entre el $45 \%$ y el $50 \%$ del total de los flujos de IED que ingresan a la región. Los países donde se destaca la predominancia de la IED en el sector servicios en relación con el total de flujos de IED son el Paraguay, la República Dominicana, el Uruguay, Honduras y el Brasil. Según datos de UNCTAD (2004), la

\footnotetext{
${ }^{1}$ La Comisión Económica para América Latina y el Caribe (CEPAL) presenta algunas estadísticas por sectores de actividad a partir de 2005 en su informe anual de IED en América Latina.
} GRÁFICO 4 América Latina (13 países): distribución sectorial de la inversión extranjera directa (IED), medias en el período 1980-2009 (En porcentajes del PIB)

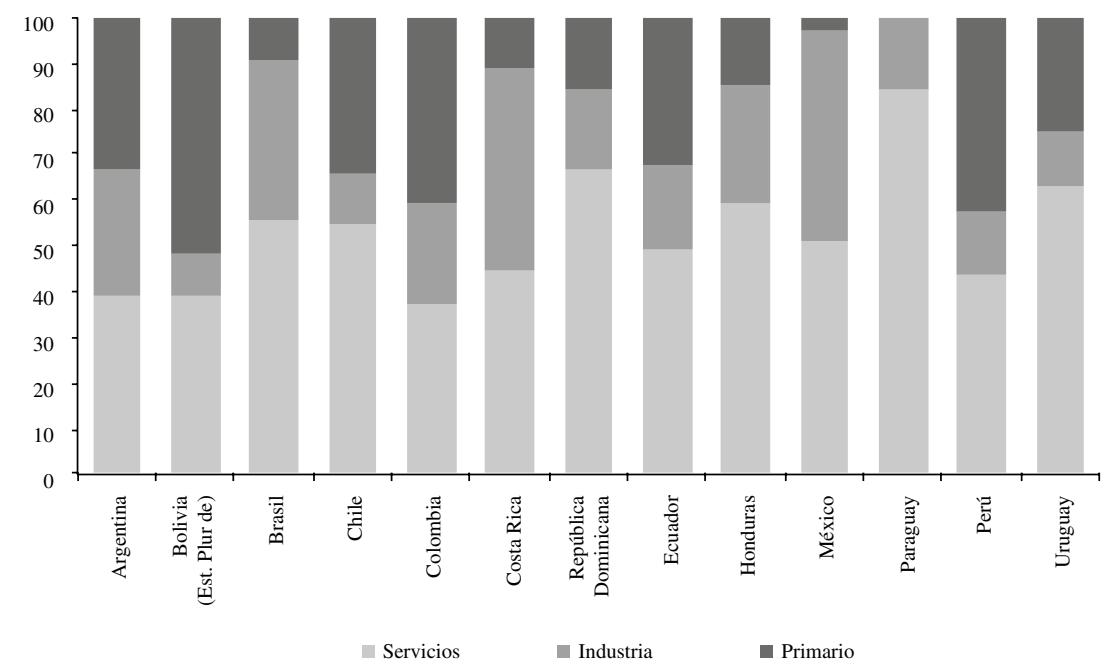

Fuente: Elaboración propia sobre la base de información de los respectivos países.

Nota: PIB: Producto interno bruto. 
IED en el sector servicios en las economías en desarrollo se ha cuadruplicado entre 1990 y 2002, y en particular en las economías de América Latina se duplicaron en el mismo período. Este último fenómeno se vincula fundamentalmente a los procesos de privatización y apertura a la inversión extranjera en el sector de las telecomunicaciones, los servicios públicos y la actividad financiera.

Como indicador de desigualdad de los ingresos se utilizó el coeficiente de Gini obtenido de la Base de datos sobre la desigualdad de los ingresos en el mundo

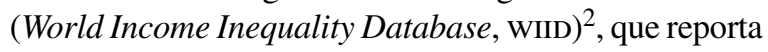
en muchos casos más de una estimación del coeficiente de Gini por año y país. Así, con el fin de utilizar datos lo más homogéneos y comparables, las observaciones fueron seleccionadas, en primer lugar, teniendo en cuenta la clasificación de calidad elaborada por el Instituto Mundial de Investigaciones de Economía del Desarrollo (UNU-WIDER), eliminando las observaciones de menor calidad (ranking $4^{3}$ ). Asimismo, se controló mediante la definición de ingreso utilizada (priorizando la de ingreso disponible) por la cobertura por área geográfica y poblacional, y también sobre la base de la fuente utilizada. Por último, para algunos países se actualizaron los datos de desigualdad por medio de la Base de datos SEDLAC $^{4}$, manteniendo el mismo criterio de selección antes mencionado. En el gráfico 5 se presentan los promedios en el coeficiente de desigualdad por país para los dos subperíodos de la muestra: 1980-1994 y 1995-2009.

En el gráfico 5 se pone de relieve que el Uruguay, Costa Rica y la Argentina se encuentran dentro del grupo de países que presentan coeficientes de desigualdad relativamente más bajos, mientras que Bolivia (Estado Plurinacional de), el Brasil y Colombia presentan los índices más elevados. Con relación a la evolución temporal, cabe destacar que, a pesar de la importante persistencia de este fenómeno, el Paraguay, el Ecuador, la Argentina y Bolivia (Estado Plurinacional de) experimentan un incremento significativo en la desigualdad durante el período analizado, mientras que México, el Brasil y el Perú son los únicos países que presentan una significativa reducción en los niveles de desigualdad. Esta distinta evolución temporal podría responder en parte a la implementación de políticas específicas en cada país.

2 De la versión WIID 2.c correspondiente a mayo de 2008, elaborada por el Instituto Mundial de Investigaciones de Economía del Desarrollo (UNU-WIDER).

${ }^{3}$ No se conocen esas observaciones.

${ }^{4}$ Base de datos socioeconómicos para América Latina y el Caribe
(SEDLAC) [en línea] http://sedlac.econo.unlp.edu.ar/esp/. GRÁFICO 5 América Latina (13 países): coeficiente de Gini por país

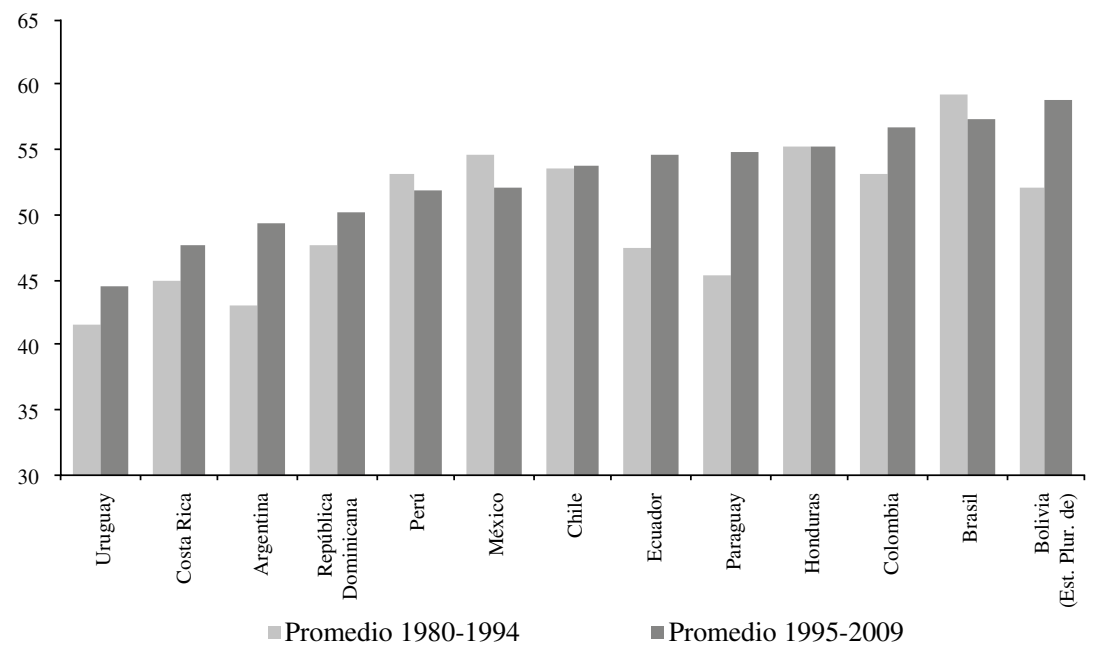

Fuente: Elaboración propia mediante información de la Base de datos sobre la desigualdad de los ingresos en el mundo (WIID) y la Base de Datos Socioeconómicos de América Latina y el Caribe (SEDlaC). 


\section{IV}

\section{Estrategia empírica}

Con el objetivo de determinar el efecto de la IED en la desigualdad de los ingresos y siguiendo los trabajos de Basu y Guariglia (2007), Choi (2006) y Tsai (1995), se estimaron dos ecuaciones, una con los datos agregados de IED, y otra que incluye la IED desagregada por sectores de actividad (primario, industria y servicios). Mediante el análisis de estos dos escenarios se procura arrojar evidencia respecto de si la IED produce diferentes efectos en la desigualdad de los ingresos según en qué sector de actividad se localice.

\section{Efecto de la IED agregada en la desigualdad de los ingresos}

El impacto de la IED a nivel agregado en la desigualdad salarial se describe en la siguiente ecuación:

$$
\text { desigualdad }_{i t}=a_{0}+a_{1} I E D_{i t}+a_{2} X_{i t}+u_{i}+e_{i t}
$$

donde la variable dependiente es una medida de la desigualdad de los ingresos (el coeficiente de Gini) para el país $i$ en el período $t$, la $I E D_{i t}$ se expresa como porcentaje del PIB en el país $i$ en el momento $t$, y $X_{i t}$ es un vector que incluye las principales variables de control. El término $u_{i}$ representa los efectos fijos por países y $e_{i t}$ corresponde al término de error.

De acuerdo con la literatura, se introdujeron las variables de control que pueden afectar tanto a la desigualdad de los ingresos como a la IED y, por lo tanto, su omisión puede causar sesgos en la estimación del impacto de la IED en la inequidad salarial. Las variables de control incluidas son: el comercio (en términos del PIB), el capital humano, el crecimiento de la población y el gasto público (en términos del PIB).

En primer lugar, con respecto a la variable explicativa comercio - que mide el comercio exterior de las economías a través del indicador del nivel de apertura comercial (la suma de las importaciones y exportaciones totales en términos del PIB)—, los datos provienen de la base World Development Indicators (WDI). La teoría tradicional del comercio, como el modelo modelo de Heckscher y Ohlin (H-O), sugiere que con el aumento del comercio la desigualdad salarial en los países en desarrollo, que suelen ser relativamente abundantes en mano de obra no calificada, tiende a disminuir. Cuanto más abierta una economía al comercio internacional, más evidente sería el efecto en la desigualdad salarial. Sin embargo, en la literatura en que se estudia la relación entre la IED y la desigualdad se predice el efecto contrario, porque se supone que la IED tiende a localizarse en los sectores con trabajadores más calificados, aumentando así la brecha salarial entre los trabajadores no calificados y los calificados (Velde, 2003).

En segundo lugar, como proxy de la variable capital humano se utilizó el promedio de años de educación secundaria en la población. Los datos proceden de la base de datos de Barro y Lee (2013). En la literatura que estudia la relación entre la desigualdad y el capital humano se expresa que un mayor nivel educativo de la población reduce la desigualdad de los ingresos (véase, por ejemplo, Castelló y Doménech, 2002). En tercer lugar, se utiliza el gasto público por ser una variable relevante en la determinación de la desigualdad de los ingresos y para controlar por el impacto de la política fiscal en dicha desigualdad. Es esperable, de acuerdo con la literatura, que un mayor gasto público redunde en una menor desigualdad de los ingresos (Atkinson y Brandolini, 2006). Finalmente, se incluye el crecimiento de la población para controlar por el tamaño de las economías. Las estadísticas descriptivas y la definición y fuente de las variables se presentan en los cuadros A.3 y A.4 del anexo.

La ecuación (1) se estimó con diferentes especificaciones para comprobar la robustez de los resultados. Primero se estimó un modelo de efectos fijos (FE) de país para controlar por la presencia de heterogeneidad inobservable entre los países y la posible omisión de variables relevantes. El problema con este tipo de modelos es que implícitamente asumen que la IED es estrictamente exógena a la desigualdad. Esto puede ser un fuerte supuesto si la IED está correlacionada con otras variables observables o no observables que afectan a la desigualdad y que no se controlan en el modelo, o si la IED es una función de la desigualdad en lugar de un factor determinante de esta. Controlando por efectos fijos de país se superaría este último problema, bajo el 
supuesto de que la correlación de la IED con el término de error es fija en el tiempo. En segundo lugar, se estimó la ecuación mediante el método de mínimos cuadrados en dos etapas (TSLS, por su siglas en inglés), con el fin de controlar por la existencia de endogeneidad entre la variable dependiente y las explicativas. Para el caso de los datos anuales se utilizaron los rezagos de las propias variables como variables instrumentales, mientras que en el caso de los datos trianuales se emplearon las variables al inicio del período como instrumento, ya que se espera que estas variables no estén correlacionadas con los errores.

Finalmente, el modelo se estimó por el método generalizado de momentos (MGM) en primeras diferencias desarrollado por Arellano y Bond (1991) y Arellano y Bover (1995). Esta técnica permite tomar en cuenta la heterogeneidad no observada en el país y, a la vez, controla por la posible existencia de endogeneidad utilizando variables rezagadas en uno o más períodos como variables instrumentales. Para los efectos de poder evaluar cuándo el modelo está bien especificado, se emplearon la prueba de Sargan, con la que se mide si el modelo está correctamente especificado y si los instrumentos son válidos, y las pruebas $\mathrm{m} 1 \mathrm{y} \mathrm{m} 2$, que miden la existencia de correlación serial de los residuos de primer y segundo orden, respectivamente (Arellano y Bond, 1991; y Blundell y Bond, 1998).

\section{V}

\section{Resultados}

En esta sección se presentan los resultados empíricos obtenidos para el panel de datos de 13 economías de América Latina durante el período 1980-2009 respecto de los dos escenarios seleccionados. En un primer escenario se presentan los resultados de la estimación del efecto de la IED a nivel agregado en la desigualdad de los ingresos. En el segundo escenario se muestra el efecto de la IED por sectores de actividad en dicha inequidad.

\section{Inversión extranjera directa (IED) y desigualdad de los ingresos}

En el cuadro 1 se observan los principales resultados de estimar la relación entre la IED y la desigualdad de los

\section{Efecto de la IED por sectores en la desigualdad}

Para investigar la relación entre la IED desagregada por sectores de actividad y la desigualdad de los ingresos se estimó la siguiente ecuación:

$$
\begin{gathered}
\text { desigualdad }_{i t}=a_{0}+a_{1} \text { IEDprim }_{i t}+a_{2} \text { IEDind }_{i t} \\
+a_{3} \text { IEDserv }_{i t}+a_{4} X_{i t}+u_{i}+e_{i t}
\end{gathered}
$$

De nuevo, la variable dependiente es una medida de la desigualdad de los ingresos para el país $i$ en el período $t$ (coeficiente de Gini); IEDprim ${ }_{i t}$, IEDind $_{i t}$ y IEDserv ${ }_{i t}$ representan la IED (como porcentaje del PIB) en el país $i$ en el momento $t$ en el sector primario, la industria manufacturera y servicios, respectivamente; $X_{i t}$ es un vector que incluye las principales variables de control correlacionadas con la desigualdad; $u_{i}$ corresponde a efectos fijos por país, y $e_{i t}$ se corresponde con el término de error.

Para estimar la ecuación (2) se utilizó la misma estrategia empírica que en la ecuación (1), y se estimó bajo tres especificaciones diferentes: FE, TSLS y MGM en primeras diferencias; y al igual que para la ecuación (1), la estrategia se replicó para los datos trianuales como método de robustez. Se incluyeron las mismas variables de control que en la primera ecuación: comercio, capital humano, gasto público y crecimiento de la población.

ingresos a nivel agregado. Las distintas estimaciones del cuadro 1 permiten confirmar la hipótesis central del presente trabajo: la relación entre la IED y la desigualdad salarial para las economías de la región es positiva y significativa en todas las especificaciones consideradas (FE, TSLS y MGM). Estos resultados están en línea con otros trabajos de la literatura (Tsai, 1995; Basu y Guariglia, 2007; Choi, 2006; Herzer, Hühne y Nunnenkamp, 2012). Así, la IED puede afectar a dos componentes de los ingresos, capital o salarios. Una posible interpretación del efecto social proviene del efecto en el salario de los trabajadores; como apunta Velde (2003), una de las principales causas de la mayor desigualdad de los ingresos generada por la IED en América Latina obedece 
CUADRO 1

Coeficiente de Gini e inversión extranjera directa (IED)

\begin{tabular}{|c|c|c|c|c|c|c|}
\hline \multirow{2}{*}{$\begin{array}{l}\text { Variable dependiente: } \\
\text { coeficiente de Gini }\end{array}$} & \multicolumn{3}{|c|}{ Datos anuales } & \multicolumn{3}{|c|}{ Datos trianuales } \\
\hline & $\begin{array}{c}\text { Efectos fijos } \\
\text { (1) }\end{array}$ & $\begin{array}{l}\text { TSLS } \\
\text { (2) }\end{array}$ & $\begin{array}{l}\text { MGM primera } \\
\text { diferencia (3) }\end{array}$ & $\begin{array}{c}\text { Efectos fijos } \\
\text { (4) }\end{array}$ & $\begin{array}{l}\text { TSLS } \\
(5)\end{array}$ & $\begin{array}{l}\text { MGM primera } \\
\text { diferencia (6) }\end{array}$ \\
\hline \multirow[t]{2}{*}{ IED } & $0,403 * * *$ & $0,544 * * *$ & $0,199 * *$ & $0,464 * * *$ & $0,449 * * *$ & $0,405 * * *$ \\
\hline & $(0,074)$ & $(0,113)$ & $(0,096)$ & $(0,113)$ & $(0,115)$ & $(0,075)$ \\
\hline Comercio & $-0,002$ & $-0,0003$ & $-0,021$ & 0,012 & 0,041 & 0,0236 \\
\hline \multirow[t]{2}{*}{ Crecimiento población } & $-1,123$ & $-1,201$ & $-1,044$ & $-1,787 *$ & $-0,654$ & 2,299 \\
\hline & $(0,827)$ & $(0,922)$ & $(0,755)$ & $(1,033)$ & $(0,947)$ & $(1,997)$ \\
\hline \multirow[t]{2}{*}{ Gasto público } & $-0,292 * * *$ & $-0,259 * *$ & $-0,133$ & $-0,269 *$ & $-0,251$ & $-0,403$ \\
\hline & $(0,083)$ & $(0,108)$ & $(0,175)$ & $(0,165)$ & $(0,212)$ & $(0,348)$ \\
\hline \multirow[t]{2}{*}{ Capital humano } & $-0,859$ & $-1,599 * *$ & $-1,133 * *$ & $-2,088 *$ & $-1,896^{* *}$ & $-1,843 *$ \\
\hline & $(0,615)$ & $(0,823)$ & $(0,512)$ & $(1,138)$ & $(0,814)$ & $(1,017)$ \\
\hline Constante & $\begin{array}{l}56,765 * * * \\
(2536)\end{array}$ & $\begin{array}{l}57,617 * * * \\
(1718)\end{array}$ & & $\begin{array}{l}59,007 * * * \\
(3562)\end{array}$ & $\begin{array}{l}54,979 * * * \\
(3269)\end{array}$ & \\
\hline Prueba de Sargan (valor $p$ ) & & & 0,520 & & & 0,124 \\
\hline Prueba m1 (valor $p$ ) & & & 0,000 & & & 0,003 \\
\hline Prueba m2 (valor $p$ ) & & & 0,625 & & & 0,181 \\
\hline Observaciones & 258 & 254 & 158 & 117 & 117 & 87 \\
\hline R2-Ajustado & 0,83 & 0,82 & & 0,82 & 0,81 & \\
\hline Países & 13 & 13 & 13 & 13 & 13 & 13 \\
\hline
\end{tabular}

Fuente: Elaboración propia.

Nota: En todas las estimaciones se incluyen los errores estándar corregidos por heterocedasticidad. La prueba de Sargan es una prueba que constata la sobreidentificación de instrumentos en los modelos MGM. Y las pruebas m1 y m2 testean si existe correlación serial de primero y segundo orden en los residuos, respectivamente.

$* * *$ Significancia al $1 \%$; ** significancia al $5 \%$; $*$ significancia al $10 \%$.

a que las empresas transnacionales tienden a localizarse en sectores de mano de obra altamente calificada, siendo estos los que absorben la mayoría de los beneficios de la IED, es decir, transferencias tecnológicas, transferencia de conocimiento y mayor productividad. Como resultado de ello, se evidencia un aumento de la brecha salarial entre trabajadores calificados y no calificados, y por tanto, un incremento de la desigualdad de los ingresos en estas economías. En la columna 1 del cuadro 1 se presenta el resultado de la estimación de efectos fijos (FE) para los datos anuales. Un cambio de una unidad porcentual en la IED para todos los países acrecienta el coeficiente de Gini en 0,40 puntos porcentuales. Este resultado es particularmente importante si se toma en cuenta la estabilidad en el tiempo que presenta dicho coeficiente. Como se mencionó en la sección anterior, con el fin de tener en cuenta la posible existencia de endogeneidad, el modelo también fue estimado por TSLS utilizando rezagos de las variables explicativas como variables instrumentales. Los resultados que se presentan en la columna 2 del cuadro 1 confirman la relación positiva y estadísticamente significativa entre la IED y la desigualdad de ingresos. Por último, se estimó el modelo de forma dinámica empleando el estimador MGM en primeras diferencias (véase la columna 3 del cuadro 1). Al igual que en las especificaciones anteriores, la IED sigue presentando signo positivo y es significativa. El orden de magnitud del coeficiente de IED es relativamente similar entre los efectos fijos y TSLS, mientras que disminuye con el estimador MGM.

Con respecto a las variables de control, la variable del gasto público tiene un efecto negativo en la desigualdad y es significativa. Este resultado está en línea con la literatura en que se estudia el impacto de la política fiscal en la desigualdad, encontrando que el mayor gasto público deriva en una reducción de la desigualdad de los ingresos (Li, Xie y Zou, 2000; Afonso, Schuknecht y Tanzi, 2010; Muinelo-Gallo y Roca Sagalés, 2011 y 2013). En la misma dirección, la variable de capital humano tiene un impacto negativo en la inequidad salarial y el resultado es robusto bajo las diferentes especificaciones. En la literatura se muestra que la mayor inversión en capital humano se traduce en una disminución de la desigualdad de los ingresos sobre todo en las economías en desarrollo (véanse Basu y Guariglia, 2007; Blomstrom y Kokko, 2003 y Castelló y Doménech, 2002). Este último resultado es relevante, dado que la educación es destacada en varios estudios como uno de 
los instrumentos más efectivos para disminuir la pobreza y la desigualdad en las economías en desarrollo y, por consiguiente, aparece como un factor relevante a tener en cuenta por los hacedores de políticas públicas en especial con carácter redistributivo.

Como medida de robustez se estimaron de nuevo los mismos modelos, pero utilizando datos trianuales (véanse las columnas 4, 5 y 6 del cuadro 1). Y, como se puede apreciar, los resultados son similares a los que se obtuvieron con datos anuales, dado que la IED tiene un signo positivo y es estadísticamente significativa en las tres especificaciones consideradas. En contraste con el caso anterior, cuando se usó el estimador TSLS se seleccionaron las variables al inicio del período como variables instrumentales, por considerarse que están poco correlacionadas con los errores. En relación con las variables de control, los resultados son también similares a los que se obtuvieron con el modelo de datos anuales. El resultado más notable es el de la variable de capital humano, ya que es el único resultado robusto en las tres especificaciones y las dos muestras, con un impacto negativo en la desigualdad. El gasto público y la variable crecimiento de la población presentan signo negativo y son estadísticamente significativos en la estimación de efectos fijos, pero no son resultados tan robustos.

\section{La IED por sectores y desigualdad de los ingresos}

En el cuadro 2 se reportan los resultados de la estimación del efecto de la IED por sectores de actividad en la desigualdad de los ingresos en América Latina para las dos muestras y bajo las tres especificaciones. Como se explicó en la sección anterior, se identificó la IED en tres grandes sectores de actividad: primario, industria manufacturera y servicios.

Como se aprecia en el cuadro 2, tanto la IED localizada en la industria manufacturera como en el sector servicios presentan signo positivo y son estadísticamente significativos. La industria manufacturera presenta el coeficiente más elevado de impacto de la IED en la desigualdad, aunque el sector servicios es el que denota las

CUADRO 2

Coeficiente de Gini e inversión extranjera directa (IED) por sectores

\begin{tabular}{|c|c|c|c|c|c|c|}
\hline \multirow{2}{*}{$\begin{array}{l}\text { Variable dependiente: } \\
\text { coeficiente de Gini }\end{array}$} & \multicolumn{3}{|c|}{ Datos anuales } & \multicolumn{3}{|c|}{ Datos trianuales } \\
\hline & $\begin{array}{c}\text { Efectos fijos } \\
\text { (1) }\end{array}$ & $\begin{array}{l}\text { TSLS } \\
(2)\end{array}$ & $\begin{array}{l}\text { MGM primera } \\
\text { diferencia (3) }\end{array}$ & $\begin{array}{c}\text { Efectos fijos } \\
\text { (4) }\end{array}$ & $\begin{array}{l}\text { TSLS } \\
(5)\end{array}$ & $\begin{array}{l}\text { MGM primera } \\
\text { diferencia (6) }\end{array}$ \\
\hline \multirow[t]{2}{*}{ IED_sectorprimario } & 0,194 & 0,171 & $-0,049$ & $0,492 * * *$ & $0,552 * * *$ & $-0,143$ \\
\hline & $(0,147)$ & $(0,135)$ & $(0,165)$ & $(0,166)$ & $(0,197)$ & $(0,249)$ \\
\hline IED_sector industria & $0,396^{*}$ & 0,422 & 0,341 & $0,901 * *$ & $2,197 * * *$ & $0,875^{* *}$ \\
\hline \multirow[t]{2}{*}{ IED_sector servicios } & $0,408 * * *$ & $0,412 * * *$ & $0,198 * *$ & $0,437 * * *$ & $0,325 * * *$ & $0,468 * * *$ \\
\hline & $(0,078)$ & $(0,079)$ & $(0,090)$ & $(0,121)$ & $(0,084)$ & $(0,160)$ \\
\hline \multirow[t]{2}{*}{ Comercio } & $0,039 * * *$ & $0,042 *$ & $-0,012$ & 0,036 & $0,057 *$ & 0,010 \\
\hline & $(0,015)$ & $(0,024)$ & $(0,035)$ & $(0,024)$ & $(0,033)$ & $(0,023)$ \\
\hline \multirow[t]{2}{*}{ Crecimiento población } & 0,875 & 0,836 & $-0,893$ & $-1,771$ & $2,388^{*}$ & 0,199 \\
\hline & $(0,883)$ & $(1,137)$ & $(0,928)$ & $(1,505)$ & $(1,439)$ & $(1,780)$ \\
\hline \multirow[t]{2}{*}{ Gasto público } & $-0,362 * * *$ & $-0,313 * * *$ & $-0,186$ & $-0,327 *$ & $-0,126$ & $-0,059$ \\
\hline & $(0,120)$ & $(0,118)$ & $(0,197)$ & $(0,179)$ & $(0,204)$ & $(0,172)$ \\
\hline Capital humano & $(0,756)$ & $(0,928)$ & $(0,566)$ & $(0,533)$ & $(0,466)$ & $(0,690)$ \\
\hline \multirow[t]{2}{*}{ Constante } & $55,626 * * *$ & $55,755 * * *$ & & $52,546 * * *$ & $75,684 * * *$ & \\
\hline & $(2903)$ & (3 455) & & $(4853)$ & $(2556)$ & \\
\hline Prueba de Sargan (valor $p$ ) & & & 0,339 & & & 0,178 \\
\hline Prueba m1 (valor $p$ ) & & & 0,000 & & & 0,017 \\
\hline Prueba m2 (valor $p)$ & & & 0,684 & & & 0,659 \\
\hline Observaciones & 171 & 170 & 121 & 74 & 68 & 57 \\
\hline R2-Ajustado & 0,87 & 0,87 & & 0,90 & 0,90 & \\
\hline Países & 13 & 13 & 13 & 13 & 13 & 13 \\
\hline
\end{tabular}

Fuente: Elaboración propia.

Nota: En todas las estimaciones se incluyen los errores estándar corregidos por heterocedasticidad. La prueba de Sargan es una prueba en que se contrasta la sobreidentificación de instrumentos en los modelos MGM. Con las pruebas m1 y m2 se comprueba si existe correlación serial de primero y segundo orden en los residuos, respectivamente.

$* * *$ Significancia al $1 \%$; ** significancia al $5 \%$; significancia al $10 \%$. 
estimaciones más robustas bajo las tres especificaciones y las dos muestras, con un coeficiente similar al registrado en la IED a nivel agregado y del orden de 0,40. Este último resultado es aún más importante para estas economías si se tienen en cuenta las tendencias observadas en la última década, donde la IED dirigida a los servicios se duplica con creces entre 1990 y 2002, tomando este sector gran relevancia como el principal receptor de esta inversión ${ }^{5}$. En tal sentido, la literatura en que se estudia el efecto de los servicios en las economías en desarrollo no es concluyente respecto del impacto que este sector puede tener en distintos aspectos de la economía, en tanto que los hacedores de políticas no están convencidos de que abrir las puertas a la IED dirigida al sector servicios tenga efectos positivos (Banga, 2005). Asimismo, es relevante destacar que por limitaciones en los datos no es posible hacer una distinción por subsectores de actividad dentro de dicho sector para todos los países. Esto implica una importante limitación en cuanto a poder capturar el efecto del sector servicios en la economía, particularmente si se considera que el sector financiero es uno de los subsectores gravitantes al explicar la evolución de la IED en América Latina, especialmente a partir de la década de 1990.

Dentro de las variables de control se destacan las variables gasto público y capital humano, que son significativas y presentan signo negativo en línea con los principales resultados de la literatura. Así, tanto la inversión en capital humano como la política fiscal aparecen como instrumentos de política económica

\footnotetext{
5 Y en particular, se transforma en un destino atractivo para la IED en los subsectores de la tecnología de turismo, de las telecomunicaciones y de la información (UNCTAD, 2004).
}

eficaces para combatir la persistente desigualdad de los ingresos presente en estas economías regionales. Este último resultado es muy robusto tanto en las distintas especificaciones como en los dos escenarios analizados: a nivel global y por sectores de actividad. Con respecto a la variable comercio, esta presenta un efecto positivo en la desigualdad y es estadísticamente significativa. Este último resultado es opuesto al de la teoría tradicional del comercio según el modelo H-O, y está en línea con la literatura donde se estudia el efecto de la IED en la desigualdad de los ingresos, que encuentra el efecto contrario, es decir, un incremento en la desigualdad de ingresos ante un crecimiento del comercio, como se explicó en la sección II.

Para finalizar, a fin de comprobar la robustez de los resultados se replicaron las estimaciones realizadas a nivel sectorial, utilizando una serie alternativa de datos de IED por sectores. Debido a que las fuentes de datos de las series de IED agregada y las series de IED por sectores son diferentes, los montos totales presentan algunas diferencias. Para intentar controlar el posible efecto provocado por dichas diferencias se reconstruyó la serie de datos por sectores, de tal manera que el sumatorio de las series por sectores coincida con el total de la IED a nivel agregado. Los resultados obtenidos mediante esas nuevas series de IED por sectores confirman que la IED en los sectores industria manufacturera y servicios incrementa la desigualdad de los ingresos, ya que se mantienen los signos y la significancia de las variables bajo las tres especificaciones consideradas y las dos muestras utilizadas 6 .

\footnotetext{
${ }^{6}$ Los resultados no se presentan en el artículo, pero se utilizan como controles de robustez de los resultados.
}

\section{VI}

\section{Conclusiones}

En este trabajo se analiza la relación de la IED con la desigualdad de los ingresos en América Latina mediante un panel de datos no balanceado para 13 economías. Uno de los principales resultados encontrados confirma la existencia de una relación positiva entre la IED y la desigualdad salarial en línea con varios de los estudios de la literatura (Basu y Guariglia, 2007; Choi, 2006; Herzer, Hühne y Nunnenkamp, 2012; Velde, 2003; Tsai,
1995). Por otra parte, cuando se analiza el efecto de la IED por sectores de actividad, los resultados evidencian que la IED realizada en el sector servicios y también en la industria manufacturera aumenta la desigualdad de los ingresos explicando el resultado anterior. Se destaca el efecto positivo de estos sectores respecto de la variable desigualdad, por tratarse de los sectores más relevantes para explicar la evolución de la IED en estas 
economías de la región en la última década según datos de la UNCTAD (2004).

Debido a las características de las economías de la región, que mayoritariamente presentan una persistente desigualdad de los ingresos en el tiempo, estos resultados revisten gran importancia desde una perspectiva de política económica, ya que aportan evidencia empírica sobre las implicancias distributivas asociadas a la IED, y en consecuencia, abren la posibilidad de que los gobiernos intenten canalizar u orientar los recursos extranjeros de tal manera que la IED alcance a los sectores sociales menos favorecidos, o en todo caso diseñen y apliquen políticas redistributivas que permitan que los beneficios provocados por la IED se extiendan y mejoren el bienestar de la población.

En resumen, este estudio supone una contribución a la literatura empírica, sobre todo al aportar evidencia respecto del efecto de la IED por sectores en la desigualdad de los ingresos en América Latina, mostrando que el sector donde la IED se lleve a cabo es relevante desde el punto de vista de sus efectos distributivos. De este modo, se pone de relieve un impacto diferencial dependiendo de si la IED se realiza en el sector servicios y en la industria manufacturera, o bien en el sector de recursos primarios.

ANEXO

CUADRO A. 1

América Latina (13 países): entrada neta de inversión extranjera directa (IED)

(En millones de dólares corrientes)

\begin{tabular}{lccr}
\hline & Promedio $1980-1994$ & Promedio 1995-2009 & Promedio 1980-2009 \\
\hline Paraguay & 42 & 120 & 89 \\
Honduras & 31 & 433 & 240 \\
Bolivia (Estado Plurinacional de) & 55 & 485 & 282 \\
Ecuador & 158 & 568 & 357 \\
Uruguay & 56 & 651 & 413 \\
Costa Rica & 121 & 524 \\
República Dominicana & 102 & 1118 & 649 \\
Perú & 286 & 2912 & 1820 \\
Colombia & 592 & 4687 & 2761 \\
Chile & 723 & 6958 & 424 \\
Argentina & 1399 & 6968 & 4302 \\
México & 3190 & 19110 & 11626 \\
Brasil & 1730 & 23863 & 14105 \\
\hline
\end{tabular}

Fuente: Elaboración propia sobre la base de Indicadores del Desarrollo Mundial (WDI). 
CUADRO A.2

Entrada neta de inversión extranjera directa (IED) por región, 1980-2009

\begin{tabular}{|c|c|c|c|c|c|c|}
\hline \multirow[b]{2}{*}{ Región } & \multicolumn{3}{|c|}{ Entrada de IED en millones de dólares } & \multicolumn{3}{|c|}{ Participación de IED en el total mundial } \\
\hline & $\begin{array}{c}\text { Promedio } \\
1980-1994\end{array}$ & $\begin{array}{c}\text { Promedio } \\
1995-2009\end{array}$ & $\begin{array}{c}\text { Promedio } \\
1980-2009\end{array}$ & $\begin{array}{c}\text { Promedio } \\
1980-1994\end{array}$ & $\begin{array}{c}\text { Promedio } \\
1995-2009\end{array}$ & $\begin{array}{c}\text { Promedio } \\
1980-2009\end{array}$ \\
\hline Economías desarrolladas & 94579 & 646398 & 370488 & $73,0 \%$ & $65,7 \%$ & $66,5 \%$ \\
\hline Economías en desarrollo & 34454 & 306436 & 170445 & $26,6 \%$ & $31,1 \%$ & $30,6 \%$ \\
\hline Oceanía & 176 & 663 & 420 & $0,1 \%$ & $0,1 \%$ & $0,1 \%$ \\
\hline África & 2915 & 23915 & 13415 & $2,3 \%$ & $2,4 \%$ & $2,4 \%$ \\
\hline Asia Occidental & 2533 & 28146 & 15339 & $2,0 \%$ & $2,9 \%$ & $2,8 \%$ \\
\hline Asia Meridional, Oriental y Sudoriental $^{\mathrm{a}}$ & 8988 & 62188 & 35588 & $7 \%$ & $6 \%$ & $6 \%$ \\
\hline América Latina y el Caribe & 9770 & 95553 & 52662 & $7,5 \%$ & $9,7 \%$ & $9,5 \%$ \\
\hline China & 10073 & 95971 & 53022 & $7,8 \%$ & $9,7 \%$ & $9,5 \%$ \\
\hline
\end{tabular}

Fuente: Elaboración propia sobre la base de datos de la Conferencia de las Naciones Unidas sobre Comercio y Desarrollo (UNCTAD).

a Sin considerar a China.

CUADRO A.3

\section{Estadísticas descriptivas}

\begin{tabular}{|c|c|c|c|c|c|}
\hline Variable & & Media & Desviación estándar & Mínimo & Máximo \\
\hline Coeficiente de Gini & $\begin{array}{l}\text { Total } \\
\text { Entre-grupos } \\
\text { Intra-grupos }\end{array}$ & 51,63 & $\begin{array}{l}5,340 \\
4,340 \\
2,883\end{array}$ & $\begin{array}{l}38,70 \\
43,23 \\
41,15\end{array}$ & $\begin{array}{l}61,88 \\
58,02 \\
58,50\end{array}$ \\
\hline IED & $\begin{array}{l}\text { Total } \\
\text { Entre-grupos } \\
\text { Intra-grupos }\end{array}$ & 2,363 & $\begin{array}{l}2,155 \\
0,855 \\
1,992\end{array}$ & $\begin{array}{r}-2,499 \\
1,112 \\
-3,388\end{array}$ & $\begin{array}{c}12,20 \\
4,431 \\
11,31\end{array}$ \\
\hline IED sector primario & $\begin{array}{l}\text { Total } \\
\text { Entre-grupos } \\
\text { Intra-grupos }\end{array}$ & 0,861 & $\begin{array}{l}1,099 \\
0,710 \\
0,872\end{array}$ & $\begin{array}{l}-1,858 \\
-0,0429 \\
-3,553\end{array}$ & $\begin{array}{l}6,295 \\
2,556 \\
6,291\end{array}$ \\
\hline IED sector industria & $\begin{array}{l}\text { Total } \\
\text { Entre-grupos } \\
\text { Intra-grupos }\end{array}$ & 0,804 & $\begin{array}{l}0,701 \\
0,347 \\
0,625\end{array}$ & $\begin{array}{l}-2,573 \\
-0,0539 \\
-1,716\end{array}$ & $\begin{array}{l}3,761 \\
1,359 \\
3,842\end{array}$ \\
\hline IED sector servicios & $\begin{array}{l}\text { Total } \\
\text { Entre-grupos } \\
\text { Intra-grupos }\end{array}$ & 1,691 & $\begin{array}{l}1,528 \\
0,632 \\
1,393\end{array}$ & $\begin{array}{r}-0,482 \\
0,958 \\
-1,439 \\
\end{array}$ & $\begin{array}{l}9,132 \\
2,647 \\
8,491\end{array}$ \\
\hline Capital humano & $\begin{array}{l}\text { Total } \\
\text { Entre-grupos } \\
\text { Intra-grupos }\end{array}$ & 1,936 & $\begin{array}{l}0,585 \\
0,451 \\
0,392\end{array}$ & $\begin{array}{l}0,708 \\
1,181 \\
1,023\end{array}$ & $\begin{array}{l}3,658 \\
2,869 \\
2,835\end{array}$ \\
\hline Crecimiento población & $\begin{array}{l}\text { Total } \\
\text { Entre-grupos } \\
\text { Intra-grupos }\end{array}$ & 1,789 & $\begin{array}{l}0,630 \\
0,539 \\
0,358\end{array}$ & $\begin{array}{r}-0,151 \\
0,482 \\
0,962\end{array}$ & $\begin{array}{l}3,133 \\
2,503 \\
2,551\end{array}$ \\
\hline Comercio & $\begin{array}{l}\text { Total } \\
\text { Entre-grupos } \\
\text { Intra-grupos }\end{array}$ & 53,42 & $\begin{array}{l}26,18 \\
22,38 \\
14,90\end{array}$ & $\begin{array}{l}11,55 \\
20,34 \\
-0,510\end{array}$ & $\begin{array}{c}136,8 \\
90,85 \\
103,5\end{array}$ \\
\hline Gasto público & $\begin{array}{l}\text { Total } \\
\text { Entre-grupos } \\
\text { Intra-grupos }\end{array}$ & 8,176 & $\begin{array}{l}4,638 \\
4,592 \\
1,412\end{array}$ & $\begin{array}{l}2,578 \\
3,346 \\
3,087\end{array}$ & $\begin{array}{l}22,59 \\
17,90 \\
13,30\end{array}$ \\
\hline
\end{tabular}

Fuente: Elaboración propia sobre la base de datos de Indicadores del Desarrollo Mundial (WDI), Base de datos sobre la desigualdad de los ingresos en el mundo (WIID), Penn World Tables, y R.J. Barro y J.W. Lee, "A new data set of educational attainment in the world, 1950-2010", Journal of Development Economics, vol. 104, Amsterdam, Elsevier, 2013.

Nota: IED: Inversión extranjera directa. 
CUADRO A.4

\section{Fuentes de datos}

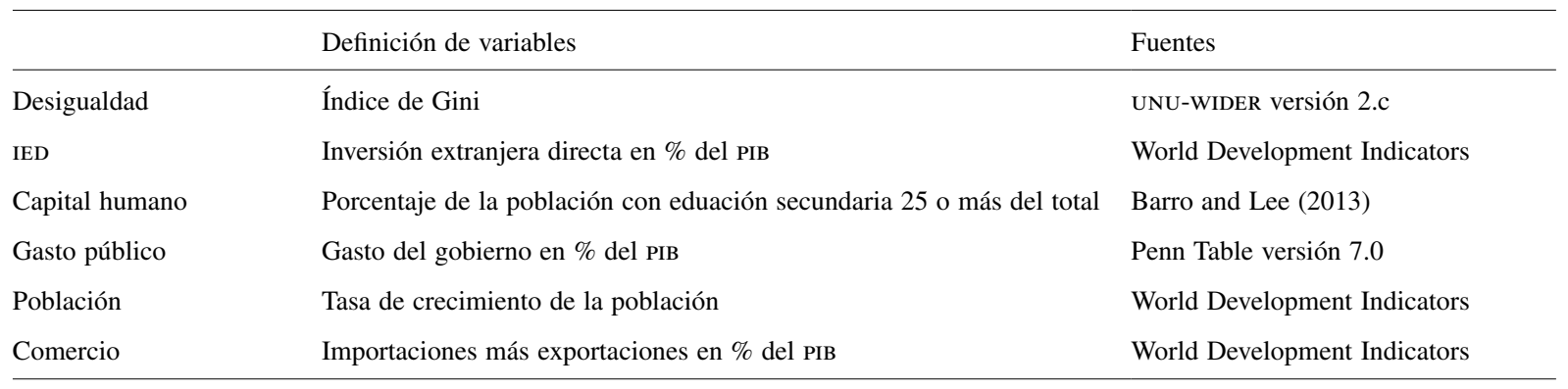

Fuente: Elaboración propia sobre la base de datos de Indicadores del Desarrollo Mundial (WDI), Base de datos sobre la desigualdad de los ingresos en el mundo (WIID), Penn World Tables, y R.J. Barro y J.W. Lee, "A new data set of educational attainment in the world, 1950-2010", Journal of Development Economics, vol. 104, Amsterdam, Elsevier, 2013.

Nota: PIB: Producto interno bruto.

CUADRO A.5

Fuentes de datos (por país)

\begin{tabular}{|c|c|}
\hline IED sectorial por país & Fuentes \\
\hline Argentina & $\begin{array}{l}\text { Instituto Nacional de Estadísticas (INDEC) } \\
\text { Dirección de Cuentas Internacionales (DNI) }\end{array}$ \\
\hline Bolivia (Estado Plurinacional de) & $\begin{array}{l}\text { Instituto Nacional de Estadísticas (INE) } \\
\text { Banco Central de Bolivia }\end{array}$ \\
\hline Brasil & Banco Central de Brasil \\
\hline Colombia & Banco Central de Colombia \\
\hline Costa Rica & Ministerio de Planificación Nacional y Política Económica (MIDEPLAN) \\
\hline Ecuador & Banco Central de Ecuador \\
\hline Honduras & Banco Central de Honduras \\
\hline México & Secretaría de Economía \\
\hline Perú & Banco Central del Perú \\
\hline República Dominicana & Banco Central de la República Dominicana \\
\hline Uruguay & Banco Central de Uruguay \\
\hline
\end{tabular}

Fuente: Elaboración propia.

Nota: IED: Inversión extrenjera directa.

\section{Bibliografía}

Aitken, B., A. Harrison y R.E. Lipsey (1996), "Wages and foreign ownership: a comparative study of Mexico, Venezuela, and the United States", Journal of International Economics, vol. 40, $\mathrm{N}^{\circ}$ 3-4, Amsterdam, Elsevier.

Afonso, A., L. Schuknecht y V. Tanzi (2010), "Income distribution determinants and public spending efficiency", Journal of Economic Inequality, vol. 8, $\mathrm{N}^{\circ} 3$, Springer.

Alarcón, D. y T. McKinley (1996), "Increasing wage inequality accompanies trade liberalisation in Mexico", The Social Challenge of the New Economic Era in Latin America, A. Berry (ed.), Toronto, Universidad de Toronto.
Arellano, M. y S. Bond (1991), "Some tests of specification for panel data: Monte Carlo evidence and an application to employment equations", Review of Economic Studies, vol. 58, № 2, Oxford University Press.

Arellano, M. y O. Bover (1995), "Another look at the instrumental variable estimation of error-component models", Journal of Econometrics, vol. 68, $\mathrm{N}^{\circ} 1$, Amsterdam, Elsevier.

Atkinson, A. y A. Brandolini (2006), "The panel-of-countries approach to explaining income inequality: an interdisciplinary research agenda", Mobility and Inequality: Frontiers of Research in Sociology and Economics, S. Morgan, D. Grusky y G. Fields (eds.), Stanford, Stanford University Press. 
Aykut, D. y S. Sayek (2007), "The role of sectoral composition of foreign direct investment on growth", Do Multinationals Feed Local Development and Growth?, L. Piscitello y G.D. Santangelo (eds.), Elsevier.

Banga, R. (2005), "Foreign direct investment in services: implications for developing countries", Asia-Pacific Trade and Investment Review, vol. 1, $\mathrm{N}^{\circ} 2$, Bangkok, Naciones Unidas.

Barro, R.J. y J.W. Lee (2013), "A new data set of educational attainment in the world, 1950-2010", Journal of Development Economics, vol. 104, Amsterdam, Elsevier.

Basu, P. y A. Guariglia (2007), "Foreign direct investment, inequality, and growth", Journal of Macroeconomics, vol. 29, $\mathrm{N}^{\circ} 4$, Amsterdam, Elsevier.

Berman, E., J. Bound y S. Machin (1998), "Implications of skillbiased technological change: international evidence", The Quarterly Journal of Economics, vol. 113, No 4, Oxford University Press.

Blomstrom, M. y A. Kokko (2003), "The economics of foreign direct investment incentives", NBER Working Paper, $\mathrm{N}^{\circ} 9489$, Cambridge, Massachusetts, National Bureau of Economic Research.

Blundell, R. y S. Bond (1998), "Initial conditions and moment restrictions in dynamic panel data models", Journal of Econometrics, vol. 87, $\mathrm{N}^{\circ} 1$, Amsterdam, Elsevier.

Borensztein, E., J. De Gregorio y J.W. Lee (1998), "How does foreign investment affect economic growth?", Journal of International Economics, vol. 45, $\mathrm{N}^{\circ} 1$, Amsterdam, Elsevier.

Castelló, A. y R. Doménech (2002), "Human capital inequality and economic growth: some new evidence", Economic Journal, vol. $112, \mathrm{~N}^{\circ} 478$, Wiley.

CEPAL (Comisión Económica para América Latina y el Caribe) (2010), Inversión extranjera directa en América Latina y el Caribe 2009 (LC/G.2447-P), Santiago.

Choi, C. (2006), "Does foreign direct investment affect domestic income inequality?", Applied Economics Letters, vol. 13, $\mathrm{N}^{\mathrm{o}} 12$, Taylor \& Francis.

Clark. D. y otros (2011), "FDI, technology spillovers, growth, and income inequality: a selective survey", Global Economy Journal, vol. 11, $\mathrm{N}^{\circ} 2$, De Gruyter.

Cornia, G.A. (2011), "Economic integration, inequality and growth: Latin America vs. the European economies in transition", Working Paper, $\mathrm{N}^{\circ} 101$, Nueva York, Departamento de Asuntos Económicos y Sociales de las Naciones Unidas.

(2004), "Trade liberalization, foreign direct investment and income inequality", Understanding Globalization, Employment and Poverty Reduction, E. Lee y M. Vivarelli (eds.), Londres, Palgrave Macmillan.

De Mello, L. (1999), "Foreign direct investment-led growth: evidence from time series and panel data", Oxford Economic Papers, vol. 51, $\mathrm{N}^{\mathrm{o}} 1$, Oxford University Press.

(1997), "Foreign direct investment in developing countries and growth: a selective survey", Journal of Development Studies, vol. 34, $\mathrm{N}^{\circ}$ 1, Taylor \& Francis.

De Vita, G. y K.S. Kyaw (2009), "Growth effects of FDI and portfolio investment flows to developing countries: a disaggregated analysis by income levels", Applied Economics Letters, vol. 16, $\mathrm{N}^{\mathrm{o}}$ 3, Taylor \& Francis.

Feenstra, R. y G.H. Hanson (1997), "Foreign direct investment and relative wages: evidence from Mexico's maquiladoras", Journal of International Economics, vol 42, $\mathrm{N}^{\circ} 3-4$, Amsterdam, Elsevier.
Herzer, D., S. Klasen y D.F. Nowak-Lehmann (2008), "In search of FDI-led growth in developing countries: the way forward", Economic Modelling, vol. 25, $\mathrm{N}^{\mathrm{o}}$ 5, Amsterdam, Elsevier.

Herzer, D., P. Hühne y P. Nunnenkamp (2012), "FDI and income inequality-evidence from Latin American economies", Working Paper, $\mathrm{N}^{\circ}$ 1791, Kiel, Instituto de Economía Mundial.

Instituto de Desarrollo de Ultramar (2002), "Foreign direct investment: who gains?", ODI Briefing Paper, Londres.

Jensen, N.M. y G. Rosas (2007), "Foreign direct investment and income inequality in Mexico, 1990-2000", International Organization, vol. 61, № 3, Cambridge, Massachusetts, The MIT Press.

Li, X. y X. Liu (2005), "Foreign direct investment and economic growth: an increasingly endogenous relationship", World Development, vol. 33, $\mathrm{N}^{\circ} 3$, Amsterdam, Elsevier.

Li, H., D. Xie y H. Zou (2000), "Dynamics of income distribution", Canadian Journal of Economics, vol. 33, $\mathrm{N}^{\circ} 4$, Canadian Economics Association.

Lipsey, R.E. y F. Sjöholm (2004), "Foreign direct investment, education and wages in Indonesian manufacturing", Journal of Development Economics, vol. 73, No 1, Amsterdam, Elsevier.

Mah, J.S. (2002), "The impact of globalization on income distribution: the Korean experience", Applied Economics Letters, vol. 9, $\mathrm{N}^{\circ} 15$, Taylor \& Francis.

Milanovic, B. (2003), "Can we discern the effect of globalization on income distribution? Evidence for household budget surveys", Policy Research Working Paper, $\mathrm{N}^{\mathrm{o}} 2876$, Washington, D.C., Banco Mundial.

Muinelo-Gallo, L. y O. Roca Sagalés (2013), "Joint determinants of fiscal policy, income inequality and economic growth", Economic Modelling, vol. 30, Amsterdam, Elsevier.

(2011), "Economic growth and inequality: the role of fiscal policy", Australian Economic Papers, vol. 50, No 2-3, Wiley Blackwell.

Nunnenkamp, P. y J. Spatz (2004), "FDI and economic growth in developing countries: how relevant are host-country and industry characteristics?", Transnational Corporations, vol. 13, $\mathrm{N}^{\circ} 3$, Ginebra, Naciones Unidas.

Sylwester, K. (2005), "Foreign direct investment, growth and income inequality in less developed countries", International Review of Applied Economics, vol. 19, No 3, Taylor \& Francis.

Tondl, G. y J.A. Fornero (2010), "Sectoral productivity and spillover effects of FDI in Latin America", FIW Working Paper Series, $\mathrm{N}^{\circ} 53$.

Tsai, P.L. (1995), "Foreign direct investment and income inequality: further evidence", World Development, vol. 23, No 3 , Amsterdam, Elsevier.

UNCTAD (Conferencia de las Naciones Unidas sobre Comercio y Desarrollo) (2011), World Investment Report 2009, Nueva York, Naciones Unidas.

(2006), World Investment Report 2006, Nueva York, Naciones Unidas.

(2004), World Investment Report 2004, Nueva York, Naciones Unidas.

Velde, W.D. te (2003), "Foreign direct investment and income inequality in Latin America: experiences and policy implications", Londres, Instituto de Desarrollo de Ultramar, inédito.

Velde, D.W. te y O. Morrissey (2004), "Foreign direct investment, skills and wage inequality in East Asia", Journal of Asia and Pacific Economies, vol. 9, $\mathrm{N}^{\circ}$ 3, Taylor \& Francis. 